\title{
RNA-seq reads of Influenza A genome appended with DNA-expression vector sequences facilitating protein transcription (18s) in Covid19 patients from Wuhan and Hong Kong - very worrying as this is unlikely to be contamination
}

Sandeep Chakraborty

\section{Letter}

Some viruses like Hepatitis $\mathrm{C}$ virus (HCV) have hijacked the human protein translation machinery [1], having by an 'internal ribosome entry site (IRES) that can autonomously bind a $40 \mathrm{~S}$ ribosomal subunit and accurately position it at the initiation codon. This binding involves both ribosomal protein and $18 \mathrm{~S}$ ribosomal RNA (rRNA) interactions' [2]. Expression vectors are designed to facilitate gene expression, by adding a promoter, a proper translation initiation sequence (a ribosomal binding site) and start/termination codon, followed by a transcription termination sequence.

\section{5 patients from Wuhan - Illumina sequencing}

Metagenome from the bronchoalveolar lavage fluid of 5 Covid19 [3-5] patients from Wuhan (Accid:PRJNA605983) shows co-infection (sequences in SI.flu.zip) with influenza A virus (3 out of 5 patients). More worryingly, these reads show the presence of $18 \mathrm{~s}$ sequences that are found in expression vectors to facilitate protein transcription (Fig. ), showing highest homology to a synthetic construct (H1N1 HA gene, Accid:KY199426.1) used in a 'digital-to-biological converter for on-demand production of biologics' [6]. Along with SARS-Cov2 infection, a few patients are also infected with Nipah [7]. Its inconceivable that a BSL-4 facility will have so many contamination. Furthermore, the sequencing was RNA-seq - while the expression vectors are DNA and thus need to be transcribed to be seen in RNA-seq data.

\section{8 patients from University of Hong Kong - Nanopore sequencing}

Metagenome from the saliva, nasopharyngeal aspirate or throat swab of 8 Covid19 patients from Hong Kong (Accid:PRJNA608242) shows the same H1N1 reads followed by the 18s sequences found in the expression vector (sequences in SI.flu.zip) in 7 patients. The Human 45S rRNA precursor transcription initiation region (Accid:K01105.1) allows more efficient transcription of the viral gene [8] (Fig ). These samples were collected in late Jan 2020.

\section{Possible origin of the Hong Kong outbreak}

One read (SRR11178053.500500) has the closest match to sequences added in 2020 from the US from various states (Fig 2) - the closest being from Oregon. These sequences (example MT303728, lineage=A(H1N1)pdm09) has been added in two studies - either "2018-2019 Influenza Surveillance among DoD Populations" or "Influenza Sequencing Activity group".

\section{References}

1. Tsukiyama-Kohara K, Iizuka N, Kohara M, Nomoto A (1992) Internal ribosome entry site within hepatitis c virus rna. Journal of virology 66: 1476-1483. 
2. Matsuda D, Mauro VP (2014) Base pairing between hepatitis c virus rna and 18s rrna is required for ires-dependent translation initiation in vivo. Proceedings of the National Academy of Sciences 111: $15385-15389$.

3. Perlman S (2020). Another decade, another coronavirus.

4. Wu F, Zhao S, Yu B, Chen Y, Wang W, et al. (2020) Complete genome characterisation of a novel coronavirus associated with severe human respiratory disease in Wuhan. China bioRxiv 24.

5. Chen L, Liu W, Zhang Q, Xu K, Ye G, et al. (2020) RNA based mNGS approach identifies a novel human coronavirus from two individual pneumonia cases in 2019 Wuhan outbreak. Emerging Microbes \& Infections 9: 313-319.

6. Boles KS, Kannan K, Gill J, Felderman M, Gouvis H, et al. (2017) Digital-to-biological converter for on-demand production of biologics. Nature biotechnology 35: 672 .

7. Chakraborty S (2020). There was a simultaneous outbreak of the zoonotic Nipah henipavirus in Wuhan - 4 out of 5 patients have the virus in Jinyintan hospital, along with SARS-Cov2, in their metagenome - which seems to have resolved by itself. doi:10.31219/osf.io/s4td6. URL osf.io/s4td6.

8. Miesfeld R, Arnheim N (1982) Identification of the in vivo and in vitro origin of transcription in human rdna. Nucleic Acids Research 10: 3933-3939. 


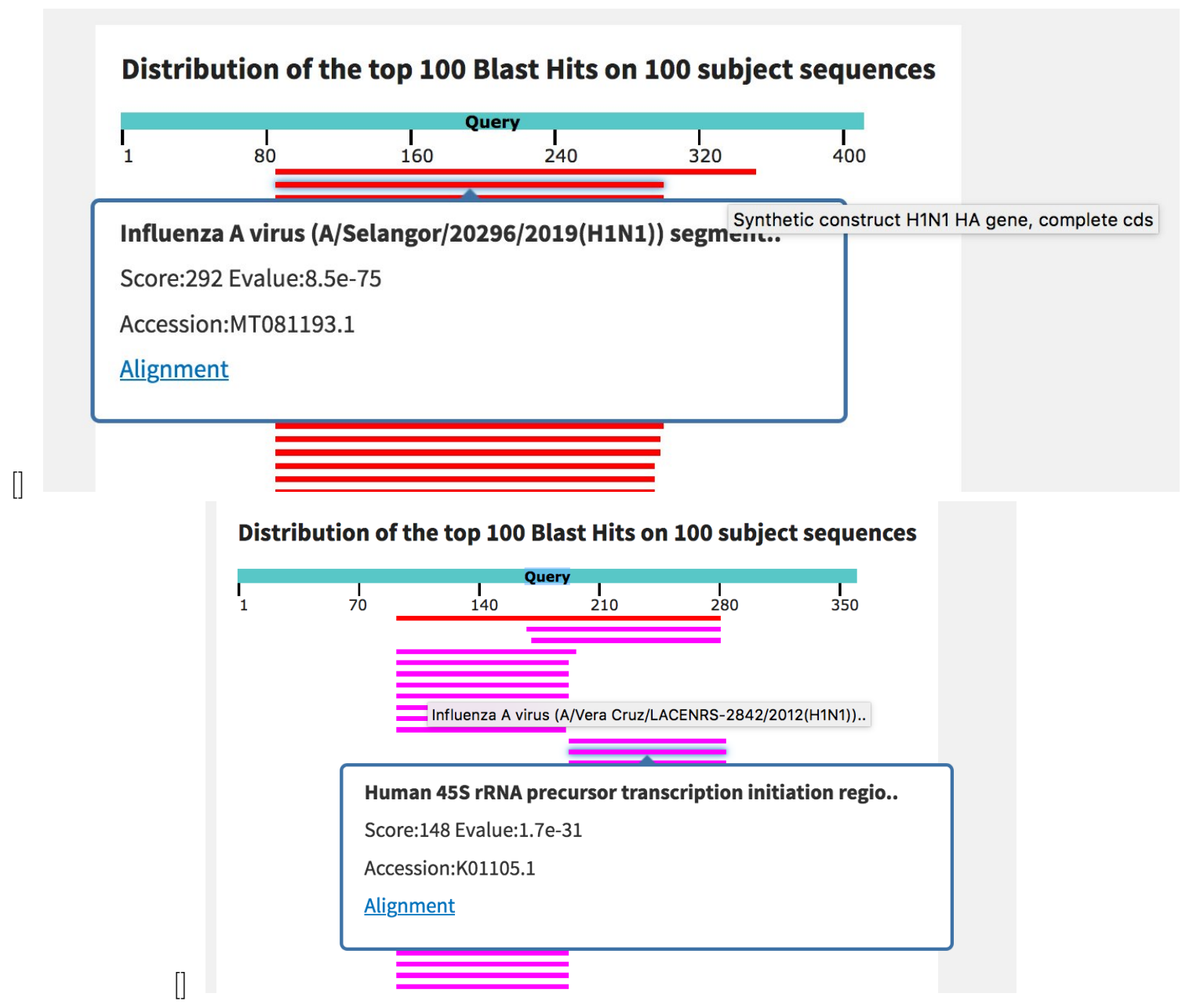

Figure 1: Reads showing the integration of the influenza gene to 18s from an expression vector: (a) Patient from Hong Kong The small extension on the first line is $18 \mathrm{~s}$ from the expression vector made to help human cells make proteins more efficiently. The $18 \mathrm{~s}$ is at the exact end of the gene, so its very unlikely to be sequencing artifacts. (b) Human 45S rRNA precursor transcription initiation region (Accid:K01105.1) allows more efficient transcription of the viral gene. 


\begin{tabular}{|c|c|c|c|c|c|c|c|}
\hline & Description & $\begin{array}{l}\text { Max } \\
\text { Score }\end{array}$ & $\begin{array}{l}\text { Total } \\
\text { Score }\end{array}$ & $\begin{array}{l}\text { Query } \\
\text { Cover }\end{array}$ & $\begin{array}{c}E \\
\text { value }\end{array}$ & $\begin{array}{l}\text { Per. } \\
\text { Ident }\end{array}$ & Accession \\
\hline $\boldsymbol{\nabla}$ & Influenza A virus (AVOregon/06/2020(H1N1)) segment 4 hemagglutinin (HA) gene, complete cds & 220 & 220 & $100 \%$ & $9 e-54$ & $100.00 \%$ & MT303728.1 \\
\hline$\nabla$ & Influenza A virus (A/swine/lowa/A02479374/2020(H1N2)) segment 4 hemagglutinin (HA) gene, complete cds & 215 & 215 & $100 \%$ & $4 e-52$ & $99.16 \%$ & MT644590.1 \\
\hline 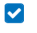 & Influenza A virus (A/swine/Wisconsin/A02479384/2020(H1N2)) segment 4 hemagglutinin (HA) gene, complete cds & 215 & 215 & $100 \%$ & $4 e-52$ & $99.16 \%$ & MT644576.1 \\
\hline$\nabla$ & Influenza A virus (A/Texas/9803/2019(H1N1)) segment 4 hemagglutinin (HA) gene, complete cds & 215 & 215 & $100 \%$ & $4 e-52$ & $99.16 \%$ & MT639869.1 \\
\hline $\boldsymbol{\nabla}$ & Influenza A virus (A/Texas/9799/2019(H1N1)) segment 4 hemagglutinin (HA) gene, complete cds & 215 & 215 & $100 \%$ & $4 e-52$ & $99.16 \%$ & MT639666.1 \\
\hline $\boldsymbol{\nabla}$ & Influenza A virus (AVEngland/9779/2019(H1N1)) segment 4 hemagglutinin (HA) gene, complete cds & 215 & 215 & $100 \%$ & $4 e-52$ & $99.16 \%$ & MT639660.1 \\
\hline $\boldsymbol{\nabla}$ & Influenza A virus (A/Colorado/9776/2019(H1N1)) segment 4 hemagglutinin (HA) gene, complete cds & 215 & 215 & $100 \%$ & $4 e-52$ & $99.16 \%$ & MT639657.1 \\
\hline $\boldsymbol{\square}$ & Influenza A virus (AWashington/9300/2019(H1N1)) segment 4 hemagglutinin (HA) gene, complete cds & 215 & 215 & $100 \%$ & $4 e-52$ & $99.16 \%$ & $\underline{\text { MT638140.1 }}$ \\
\hline$\nabla$ & Influenza A virus (A/Ohio/9268/2019(H1N1)) segment 4 hemagglutinin (HA) gene, complete cds & 215 & 215 & $100 \%$ & $4 e-52$ & $99.16 \%$ & MT638135.1 \\
\hline$\nabla$ & Influenza A virus (Alttaly/9231/2019(H1N1)) segment 4 hemagglutinin (HA) gene, complete cds & 215 & 215 & $100 \%$ & $4 e-52$ & $99.16 \%$ & MT638116.1 \\
\hline $\boldsymbol{\nabla}$ & Influenza A virus (A/Germany/9212/2019(H1N1)) segment 4 hemagglutinin (HA) gene, complete cds & 215 & 215 & $100 \%$ & $4 e-52$ & $99.16 \%$ & MT638111.1 \\
\hline $\boldsymbol{v}$ & Influenza A virus (A/Germany/9206/2019(H1N1)) segment 4 hemagglutinin (HA) gene, complete cds & 215 & 215 & $100 \%$ & $4 \mathrm{e}-52$ & $99.16 \%$ & MT638110.1 \\
\hline$\nabla$ & Influenza A virus (A/Belgium/9175/2019(H1N1)) segment 4 hemagglutinin (HA) gene, complete cds & 215 & 215 & $100 \%$ & $4 e-52$ & $99.16 \%$ & MT638100.1 \\
\hline
\end{tabular}

Figure 2: Possible origin of the virus in HK One read (SRR11178053.500500) has the closest match to sequences added in 2020 from the US from various states (Fig ??) - the closest being from Oregon. 